\title{
Detection of coke deposits on a fixed-bed catalyst by a contactless microwave method: first measurements
}

\author{
Dieter Rauch $^{1,2}$, Peter Fremerey ${ }^{1,2}$, Andreas Jess ${ }^{2}$, Ralf Moos ${ }^{1}$ \\ ${ }^{1}$ Department of Functional Materials, University of Bayreuth, 95440 Bayreuth, \\ Universitätsstraße 30, Germany, Dieter.Rauch@uni-bayreuth.de \\ ${ }^{2}$ Department of Chemical Engineering, University of Bayreuth, 95440 Bayreuth, \\ Universitätsstraße 30, Germany
}

\begin{abstract}
:
The aim of this work is to investigate a novel contactless in-operando microwave-based measurement technique for the detection of coke deposits in industrial fixed-bed catalysts. Their steel reactors serve as cavity resonators for microwaves. Due to coke loading, the electrical conductivity of the catalyst pellets increases strongly. This leads to changes in the resonance behavior that are mirrored by the scattering parameters. The regeneration of the coked catalyst by coke burn-off is examined as well.
\end{abstract}

Key words: cavity perturbation, coke deposits, contactless in-operation, cavity resonator, resonance behavior

\section{Introduction}

In the chemical industry, porous heterogeneous fixed-bed catalysts are widely used to enhance the rate of chemical reactions and to influence the product selectivity. When organic compounds are used as reagents, carbonaceous deposits are frequently produced on the catalyst surface in unwanted side reactions. Depending on the reagents, the catalysts, and the reaction conditions, the coke formation takes a few seconds up to years and the chemical composition and structure varies widely. These coke deposits lead to catalyst deactivation owing to fouling. They block the active sites of the catalyst and hinder the mass transfer into the pores [1]. Therefore, the coke has to be removed by burn-off with oxygen to restore the full catalytic activity.

Currently the coke loading is usually determined ex-situ via probe sampling and a subsequent thermogravimetric analysis [2, 3]. Hence, at the moment there is no way to monitor the catalyst state continuously, which would be beneficial for efficiency and safety reasons. Therefore a contactless microwavebased measurement technique is examined in this work to monitor the coking and decoking process directly and in-situ. This technique has successfully been tested on automotive exhaust gas after treatment systems like three-way catalysts [4], ammonia SCR-catalysts [5], diesel particulate filters [6] and Lean $\mathrm{NO}_{x}$ traps [7, 8].

\section{Experimental setup}

The microwave method is applied to an existing tubular lab-scale steel reactor. Influences of the reactor layout on this measurement technique are studied using simulation software. $105 \mathrm{~g}$ alumina catalyst pellets are used in all tests. The catalyst is fixed in the middle of the reactor by non-conductive ceramic trays with many small holes to enable the gas flow to pass through. The reactor is heated up to $560{ }^{\circ} \mathrm{C}$. For the coking experiments, $33 \%$ propane (carbon/coke source) mixed with $\mathrm{N}_{2}$ is used as feed gas at a total flow rate of $30 \mathrm{l} / \mathrm{h}$. The regeneration of the catalyst is performed at a total flow rate of $15 \mathrm{l} / \mathrm{h}$ with $2 \% \mathrm{O}_{2}$ in $\mathrm{N}_{2}$. Such coking conditions have already been used for studies that determine the amount of formed coke in industrial reactors by conductometric sensors [9]. Therefore, the coke formation rate is well-known.

To excite the electromagnetic waves, two coaxial antennas (waveguide feeds) were manufactured and mounted up- and downstream of the fixed-bed. Both antennas are connected to a vector network analyzer via coaxial cables. With the network analyzer, the scattering parameters (S-parameters) of the resonator are measured between 1 and $20 \mathrm{GHz}$. By plotting the magnitude of the Sparameters in $\mathrm{dB}$ over frequency, the resonance frequencies can be evaluated at the local extrema of the curves. Moreover, the attenuation of the electromagnetic waves can 

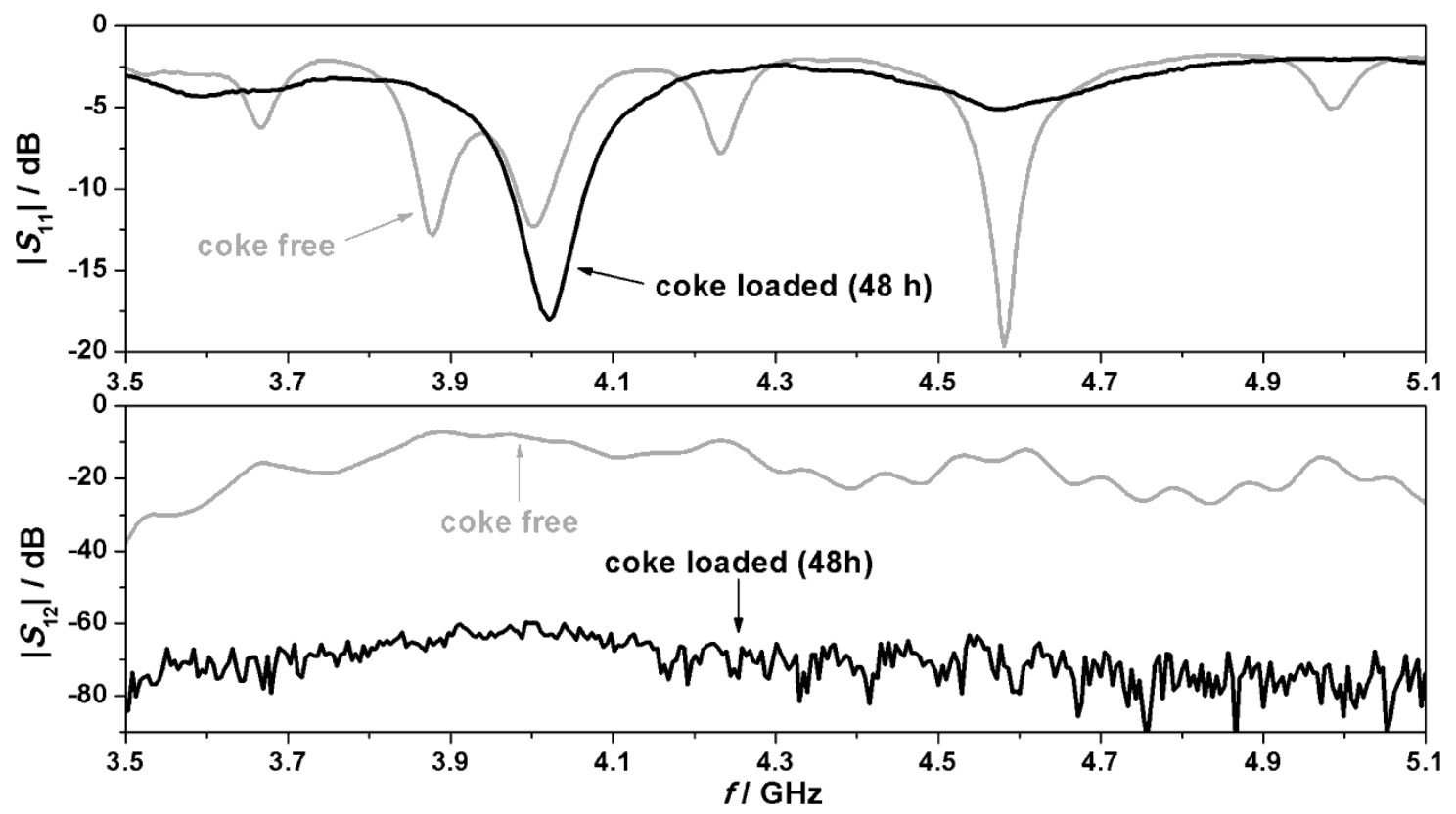

Fig. 1. Resonance spectra for the coke-free (gray) and the coke loaded (about $10 \mathrm{~g}_{\mathrm{C}} / 100 \mathrm{~g}_{\text {cat, }}$ black) fixed-bed. In the upper graph, the reflection parameter $\left|S_{11}\right|$ is shown over frequency. The lower graph displays the transmission parameter $\left|S_{12}\right|$.

be observed. Further details of this also called cavity perturbation method can be found in [10]. The coke deposits change the electrical properties of the resonator filling, especially the conductivity [11]. These alterations lead to noticeable changes of the resonant frequencies and the attenuation of the waves.

\section{Measurement results}

To validate the resonance behavior of the reactor without catalyst, a simulation model has been created using COMSOL Multiphysics. Therewith, the S-parameters are computed. The obtained spectra suit the measurement results quite well. By this way, it is also possible to investigate the influences of various geometric aspects on the measurement technique. The simulation results also match analytical calculations.

Static measurements of the reactor reveal an extensive temperature dependence of this method. The resonance frequencies shift by 1.5 to $2 \%$ due to a temperature difference of $540{ }^{\circ} \mathrm{C}$

Figure 1 shows the measured values of the reflection parameter $\left|S_{11}\right|$ and the transmission parameter $\left|S_{12}\right|$ in the reactor over frequency in the range from 3.5 to $5.1 \mathrm{GHz}$. The gray curves were taken at the beginning of the measurement, when the catalyst particles are coke free. The black curves show the state after 48 hours of coking, when the particles have reached a carbon load of about $10 \mathrm{gc}_{\mathrm{c}} / 100 \mathrm{~g}_{\text {cat }}$. Both parameters, $\left|S_{11}\right|$ and $\left|S_{12}\right|$, reveal an obvious dependence on the coke loading. The reflection parameter curve in the coke-free state shows several peaks, each for a distinct mode. When the particles are fully coke loaded, only one peak is clearly visible. The transmission parameter does not exhibit the separate resonance frequencies distinctly, but the parameter values decrease by approximately $45 \mathrm{~dB}$ with coke loading.

To illustrate the effects of the coke deposits on $\left|S_{11}\right|$ in detail, figure 2 shows one peak of the spectrum for different coking times. In the first four hours no changes in the spectrum were observed. Thereafter, the resonance frequency noticeably shifts towards lower values and the amplitude decreases with increasing time and accordingly increasing coke loading. The last curve shown was taken after 24 hours of coking which corresponds to a coke load of approximately $5 \mathrm{gc} / 100 \mathrm{~g}_{\text {cat }}$. Thereafter, no further change of the resonance frequency can be observed.

The influence of the coke loading on the transmission parameter $\left|S_{12}\right|$ is examined particularly as well. Therefore single peaks of $\left|S_{12}\right|$ are tracked over the coking time. In figure 3 the attenuation of the peak occurring at $5.05 \mathrm{GHz}$ is plotted over the coking time. After an initial time of 5 hours, during which the amount of coke on the catalyst is too small to be acquired with this measurement technique, the attenuation starts to increase. After 42 hours of coking, the final value of $-68 \mathrm{~dB}$ is reached. Thereafter, the signal is overlaid with noise, probably caused by the experimental setup. 


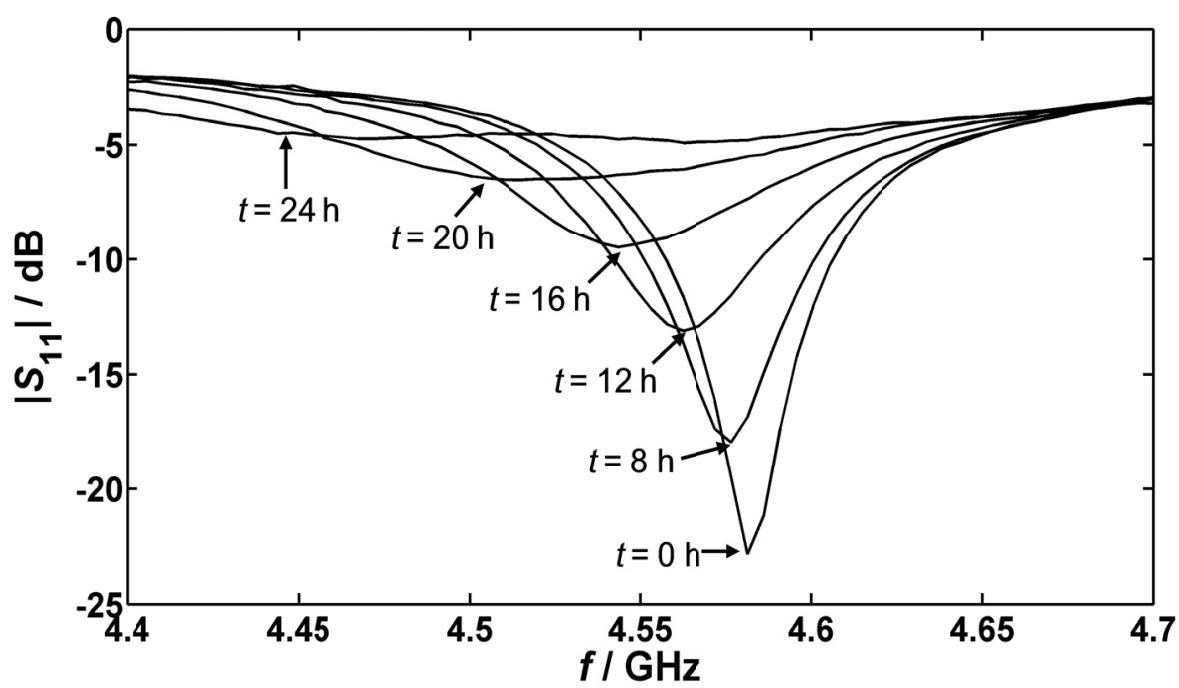

Fig. 2. A single resonance mode of $\left|S_{11}\right|$ for different coking times.

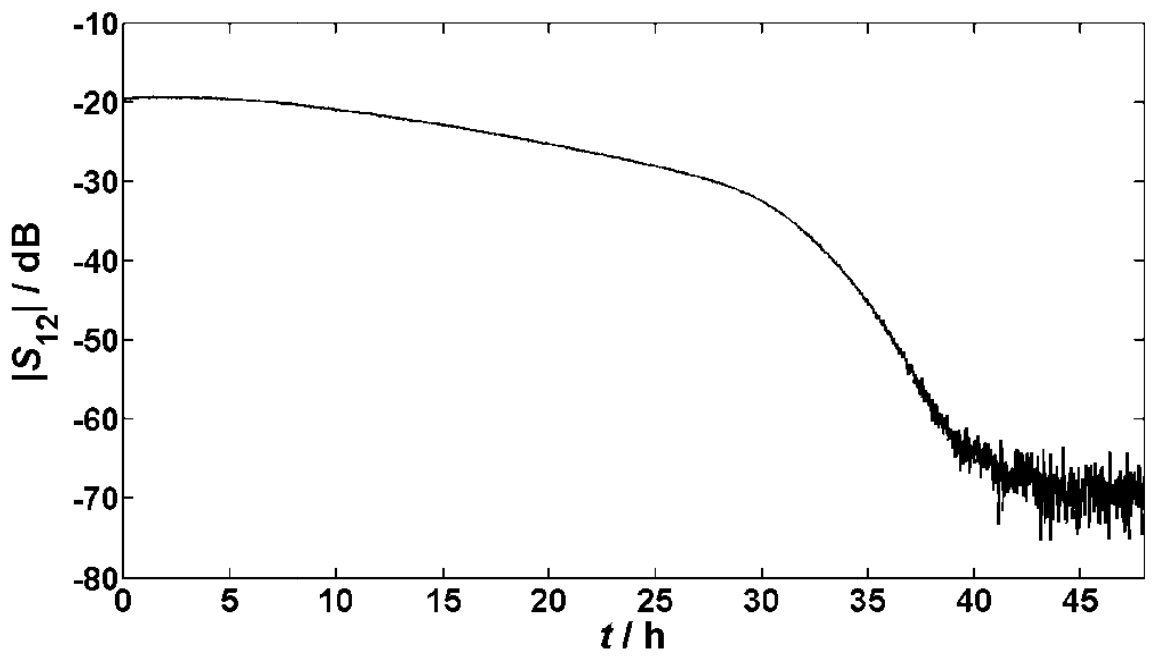

Fig. 3. Attenuation of a single peak of $\left|\mathrm{S}_{12}\right|$ over the coking time.

Hence, using the transmission parameter $\left|S_{12}\right|$ the coking process can be observed for a longer time and therewith a larger amount of coke can be detected, compared to the reflection parameter $\left|S_{11}\right|$. An obvious reason why the level of coke loading can only be monitored to a certain amount is that the electrical conductivity of the catalyst exceeds a limit above which no wave propagation through the fixed-bed is possible.

The deactivation of the catalyst due to the carbonaceous deposits is reversible. The coke can be burnt off with oxygen. During the regeneration of the coked fixed-bed in this way the resonance behavior changes in reversed order. The spectra at the end of the burn-off coincide with the ones before coke loading. This finding proves that the coke is removed completely.

\section{Acknowledgements}

The authors A.J. and R.M. are indebted to the German Research Foundation (DFG) for financial support (Je 257//12-2, Mo 1060/5-2) respectively.

\section{References}

[1] C. Kern, A. Jess, Verkokung und Koksabbrand in heterogenen Katalysatoren, Chemie Ingenieur Technik 78, 8, 1033-1048 (2006); doi: $10.1002 /$ cite. 200600051

[2] N. Müller, C. Kern, R. Moos, A. Jess, Direct detection of coking and regeneration of single particles and fixed bed reactors by electrical sensors, Applied Catalysis A: General, 382, 254262 (2010); doi: 10.1016/j.apcata.2010.05.001

[3] S. Ordonez, H. Sastre, F.V. Diez, Thermogravimetric determination of coke deposits on alumina-supported noble metal catalysts used as hydrodechlorination catalysts, Thermochimica Acta 379, 25-34 (2001); doi: 10.1016/S0040-6031(101)00598-6 
[4] R. Moos, M. Wedemann, M. Spörl, S. Reiß, G. Fischerauer, Direct Catalyst Monitoring by Electrical Means: An Overview on Promising Novel Principles, Topics in Catalysis, 52, 20352040 (2009); doi: 10.1007/s11244-009-9399-6

[5] S. Reiß, D. Schönauer, G. Hagen, G. Fischerauer, R. Moos, Monitoring the ammonia loading of zeolite-based ammonia SCR catalysts by a microwave method, Chemical Engineering and Technology, 34, 791-796 (2011); doi: $10.1002 /$ ceat. 201000546

[6] G. Fischerauer, M. Förster, R. Moos, Sensing the Soot Load in Automotive Diesel Particulate Filters by Microwave Methods, Measurement Science and Technology, 21, 035108 (2010); doi:10.1088/0957-0233/21/3/035108

[7] P. Fremerey, S. Reiß, A. Geupel, G. Fischerauer, R. Moos, Determination of the $\mathrm{NO}_{x}$ Loading of an Automotive Lean $\mathrm{NO}_{x}$ Trap by Directly Monitoring the Electrical Properties of the Catalyst Material Itself, Sensors, 11, 8261-8280 (2011); doi: $10.3390 / \mathrm{s} 110908261$

[8] R. Moos, Catalysts as Sensors - A Promising Novel Approach in Automotive Exhaust Gas Aftertreatment, Sensors, 10, 6773-6787 (2010); doi: $10.3390 / \mathrm{s} 100706773$

[9] N. Müller, A. Jess, R. Moos, Direct detection of coke deposits on fixed bed catalysts by electrical sensors, Sensors and Actuators B: Chemical, 144, 437-442 (2010); doi: 10.1016/j.snb.2009.03.008

[10] G. Fischerauer, M. Spörl, A. Gollwitzer, M. Wedemann, R. Moos, Catalyst State Observation via the Perturbation of a Microwave Cavity Resonator, Frequenz, 62, 180-184 (2008); doi: 10.1515/FREQ.2008.62.7-8.180

[11] N. Müller, R. Moos, A. Jess, In-situ monitoring of coke deposits during coking and regeneration of solid catalysts by electrical impedance-based sensors, Chemical Engineering and Technology, 33, 103-112 (2010); doi: 10.1002/ceat.200900380 